\section{HEALTH CARE SEEKING BEHAVIOUR IN URBAN INDUSTRIAL AREAS: IMPLICATIONS FOR THE DESIGN OF HEALTH SERVICES}

Rubana Islam, Syed Jafar Raza Rizvi, Alayne M Adams. Centre for Equity and Health Systems, icddr, Dhaka, Bangladesh

10.1136/bmjopen-2015-forum2015abstracts.90

Background Economic opportunities created by industrialization, prominent in urban spaces, attract a large number of migrant workers. Many of these migrants end up in poor urban settlements characterized by crowding, lack of safe water and adequate sanitation, and insecurity. Health hazards associated with these challenges, coupled with poor workplace conditions and inadequate health services, pose many health risks for the workers in low and middle income countries like Bangladesh. Given historical evidence that the health of the working class determined the net effect of industrialization on population health, it is important to highlight the health needs and choices of this emergent population and understand impediments to healthcare.

Objectives This paper explores the healthcare need and identify the factors that influence the healthcare seeking behavior of the working poor in an industrialized area.

Methods A community based cross sectional survey was performed in Tongi, Bangladesh an epicenter of garment industry. Through 2-staged cluster sampling 969 interviews among men and women of all ages were conducted. Reported health problems were categorized using ICPC-2 code. Univariate analytical approach was applied to describe health problems and associated costs, a bivariate approach explored the association between health care choices for acute and chronic health problems and select factors reported in literature, and finally logistic regression is applied to isolate the effects of individual factors.

Result There was a high prevalence of unspecified symptoms like fever and cough. Preliminary analysis showed $87 \%$ men and $77 \%$ women respondents sought treatment. Only a half of all respondents sought care from qualified doctors and majority relied heavily on private providers. Formal channels were 
consulted most for under 5 children and those aged above 65 years. Choices were predominantly influenced by proximity and cost of providers. Very few of the employed reported utilization of health services at their workplace.

Conclusion The healthcare seeking pattern that emerged in this study is not much different than what has been documented for rural areas- they depend heavily on the informal private providers and quality of care is trumped by cost and proximity of services. What stops them from consulting with their workplace health providers remains a matter of further exploration through qualitative interviews.

Urban residents are assumed to have better healthcare access and behavior given the high density of health service providers compared to rural sites. However, the working poor in urban areas has their own limitations and priorities to attend when faced with health problems. Thus far they have been a neglected group when it comes to urban health planning. Study results point to possible strategies to encourage better utilization of formal care by this growing but marginalized working population. 